\title{
ON THE ABSOLUTE CONTINUITY OF THE LIMIT RANDOM VARIABLE IN THE SUPERCRITICAL GALTON-WATSON BRANCHING PROCESS
}

\author{
KRISHNA B. ATHREYA
}

\begin{abstract}
Let $\left\{Z_{n}: n \geqq 0\right\}$ be a simple Galton-Watson branching process with offspring distribution $\left\{p_{j}\right\}$ satisying $1<\sum j p_{j}$ $<\infty$. It is known that there exist constants $C_{n}$ such that $W_{n} \equiv Z_{n} C_{n}$ converges with probability one to a nondegenerate limit random variable $W$. Here we show that this $W$ is always absolutely continuous on $(0, \infty)$.
\end{abstract}

Let $\left\{Z_{n}: n \geqq 0\right\}$ be a Galton-Watson branching process with offspring probability generating function $f(s) \equiv \sum_{j=0}^{\infty} p_{j} s^{j}$. Assume $P\left(Z_{0}=1\right)=1,\left\{p_{j}\right\}$ is nondegenerate, $1<m \equiv \sum j p_{j}<\infty$ and $p_{0}=0$. Seneta [5] and Heyde [3] have shown that there always exists a sequence of constants $C_{n} \rightarrow \infty, C_{n}^{-1} C_{n+1} \rightarrow m$ such that $W_{n} \equiv Z_{n} C_{n}^{-1}$ converges with probability one to a nondegenerate random variable $W$. Kesten and Stigum [4] had shown earlier that when $\sum p_{j} j \log j$ $<\infty, C_{n}$ may be taken as $m^{n}$ and in this case the limit $W$ has an absolutely continuous distribution on $(0, \infty)$. Their main tool was to show that if $\phi$ is the characteristic function of $W$ then $\phi^{\prime}$, the derivative, is integrable. This will fail when $\sum p_{j} j \log j=\infty$ since in this case $E W=\infty$ and the existence of $\phi^{\prime}$ is not guaranteed, let alone its integrability. In this paper we shall present a simple idea to show that $W$ is always absolutely continuous.

If $\phi(i t) \equiv E\left(e^{i t W}\right)$ is the characteristic function of $W$ then it is easily seen using the fact $C_{n}^{-1} C_{n+1} \rightarrow m$ that $\phi$ satisfies the so-called Abel's functional equation.

$$
\phi(i t)=f(\phi(i t / m)) .
$$

The equation and the nondegeneracy of $W$ ensures that $|\phi(i t)|<1$ for $t \neq 0$. We exploit this to get a rate of decay for $\phi(i t)$ as $t \rightarrow \infty$. First we recall the following about the rate of convergence of $f_{n}(x)$ for $|x|<1$.

Lemma 1. If $p_{1}>0$ then $p_{1}^{-n} f_{n}(x) \uparrow Q(x)<\infty$ for $0 \leqq x<1$. If $p_{1}=0$ then for any $\epsilon>0, \lim _{n \rightarrow \infty} \epsilon^{-n} f_{n}(x)=0$ for $0 \leqq x \leqq 1$.

Received by the editors November 23, 1970.

AMS 1969 subject classifications. Primary 6067; Secondary 6030.

Key words and phrases. Galton-Watson branching process, supercritical, absolute continuity, limit random variable.

Copyright @ 1971, American Mathematical Society 
For a proof see [1].

Let $0<\delta \leqq \infty$ be defined by $p_{1}=m^{-\delta}$.

LeMma 2. If $p_{1}>0$ then $\sup _{t}|t|^{\delta}|\phi(i t)|<\infty$. If $p_{1}=0$ then, for any $\theta>0, \sup _{t}|t|^{\theta}|\phi(i t)|<\infty$.

Proof. By continuity $\beta \equiv \sup _{1 \leq|t| \leq m}|\phi(i t)|<1$. Iterating (1) we get $\phi\left(i m^{n} t\right)=f_{n}(\phi(i t))$ and hence $\sup _{1 \leq|t| \leq m}\left|\phi\left(i m^{n} t\right)\right| \leqq f_{n}(\beta)$. Now use Lemma 1 to complete the proof. q.e.d.

If $\delta>1$ then Lemma 2 says that $\phi$ is integrable and so $W$ is absolutely continuous on $(0, \infty)$. In fact, it has a uniformly continuous density function. Assume for the rest of the paper that $\delta \leqq 1$. Let $k$ be the smallest integer such that $k \delta>1$.

LEMmA 3. For all $r \geqq k$, the $r$ fold convolution $S_{r}$ of $W$ is absolutely continuous on $(0, \infty)$ and has a uniformly continuous density.

Proof. The characteristic function of $S_{k}$ is $\phi^{k}(i t)$ and, by Lemma 2, $\sup _{t}|t|^{k \delta}\left|\phi^{k}(i t)\right|<\infty$. But $k \delta>1$ and so $\phi^{k}$ and $\phi^{r}$ for $r \geqq k$ are integrable. q.e.d.

The following is a key step.

Lemma 4. Let $W_{j}, j=0,1,2, \cdots$, be independent random variables with the same distribution as $W$. Assume further that the $W_{j}$ sequence is independent of our Galton-Watson branching process $\left\{Z_{n}\right\}$. Then $W_{0}$ has the same distribution as $\left(1 / m^{n}\right) \sum_{j=1}^{Z_{n}} W_{j}$ for each $n$.

Proof. The characteristic functions of the above two random variables are respectively $\phi(i t)$ and $f_{n}\left(\phi\left(i t / m^{n}\right)\right)$. They are equal for all $t$ as can be seen by iterating (1) $n$ times. q.e.d.

We shall now show that the absolute continuity of $S_{k}$ for all large $k$ implies the same for $W$.

Lemma 5. Let $E$ be a Borel set with Lebesgue measure zero. Then, $P(W \in E)=0$.

Proof. From Lemma 4 we see that

$$
\begin{aligned}
P\left(W_{0} \in E\right) & =\sum_{r=1}^{\infty} P\left(Z_{n}=r, \frac{1}{m^{n}} \sum_{j=1}^{Z_{n}} W_{j} \in E\right) \\
& =\sum_{r=1}^{\infty} P\left(Z_{n}=r, \frac{1}{m^{n}} \sum_{j=1}^{r} W_{j} \in E\right) \\
& =\sum_{r=1}^{\infty} P\left(Z_{n}=r\right) P\left(\sum_{j=1}^{r} W_{j} \in m^{n} E\right)
\end{aligned}
$$

(by independence of $\left\{Z_{n}\right\}$ and $\left\{W_{j}\right\}$ ). 
But by Lemma 3 , for $r \geqq k, P\left(\sum_{j=1}^{r} W_{j} \in m^{n} E\right)=0$. Thus $P\left(W_{0} \in E\right)$ $\leqq P\left(Z_{n}<k\right)$ for each $n$. Clearly, $P\left(Z_{n}<k\right) \rightarrow 0$ as $n \rightarrow \infty$ for each fixed k. q.e.d.

Lemma 5 does not assert that the density $w(x)$ of $W$ is continuous. ${ }^{1}$ If this is the case then the argument in [2] yields the conclusion that $w(x)>0$ for all $x>0$. One can perhaps then prove a local limit theorem for the $\sum p_{j} j \log j=\infty$ case in analogy with the results of [2].

\section{REFERENCES}

1. K. B. Athreya and P. Ney, Branching processes, Springer-Verlag, Berlin (forthcoming).

2. - The local limit theorem and some related aspects of the super-critical branching process, Trans. Amer. Math. Soc. 152 (1970), 233-251.

3. C. C. Heyde, Extension of a result of Seneta for the super-critical Galton-Watson process, Ann. Math. Statist. 41 (1970), 739-742. MR 40 \#8136.

4. H. Kesten and B. P. Stigum, $A$ limit theorem for multidimensional Galton-Watson processes, Ann. Math. Statist. 37 (1966), 1211-1223. MR 33 \#6707.

5. E. Seneta, On recent theorems concerning the supercritical Galton-Watson process, Ann. Math. Statist. 39 (1968), 2098-2102. MR 38 \#2847.

University of Wisconsin, Madison, Wisconsin 53706

${ }^{1}$ ADDED IN PROOF. S. Dubuc has just shown that $w(x)$ is continuous by using different techniques. He has many more results on this topic. See S. Dubuc, Ann. Inst. Fourier (Grenoble) 21 (1971), 171-251. 